\title{
The isotropic-to-nematic phase transition in hard helices: Theory and simulation
}

\author{
Elisa Frezza, ${ }^{1}$ Alberta Ferrarini, ${ }^{1, a)}$ Hima Bindu Kolli, ${ }^{2}$ Achille Giacometti, ${ }^{2, b)}$ \\ and Giorgio Cinacchi ${ }^{3, c)}$ \\ ${ }^{1}$ Dipartimento di Scienze Chimiche, Università di Padova, via Marzolo 1, 35131 Padova, Italy \\ ${ }^{2}$ Dipartimento di Scienze Molecolari e Nanosistemi, Università Ca' Foscari di Venezia, Dorsoduro 2137, \\ 30123 Venezia, Italy \\ ${ }^{3}$ Departamento de Física Teórica de la Materia Condensada, Universidad Autónoma de Madrid, \\ Campus de Cantoblanco, 28049 Madrid, Spain
}

(Received 21 December 2012; accepted 3 April 2013; published online 29 April 2013)

\begin{abstract}
We investigate the isotropic-to-nematic phase transition in systems of hard helical particles, using Onsager theory and Monte Carlo computer simulations. Motivation of this work resides in the ubiquity of the helical shape motif in many natural and synthetic polymers, as well as in the well known importance that the details of size and shape have in determining the phase behaviour and properties of (soft) condensed matter systems. We discuss the differences with the corresponding spherocylinder phase diagram and find that the helix parameters affect the phase behaviour and the existence of the nematic phase. We find that for high helicity Onsager theory significantly departs from numerical simulations even when a modified form of the Parsons-Lee rescaling is included to account for the non-convexity of particles. ( 2013 AIP Publishing LLC. [http://dx.doi.org/10.1063/1.4802005]
\end{abstract}

\section{INTRODUCTION}

In the physics of fluids, simple and complex, it is well established that size and shape of particles, either molecular or colloidal, play a key role in determining thermodynamics, structure, and dynamics (e.g., Refs. 1 and 2). Hard body particles, interacting with each other through steep repulsive potentials only, can thus be viewed as elementary models to understand the phase behaviour and properties of physical systems.

While the first example of phase transition driven by purely steric interactions is undoubtedly the fluid-to-crystal phase transition in hard spheres, ${ }^{3}$ the isotropic-to-nematic liquid crystal (IN) phase transition in hard slender rods predicted by Onsager ${ }^{4}$ paved the way for an entirely new field. Although Onsager theory was originally motivated by the observation of a nematic liquid crystal phase in suspensions of inorganic and biological rod-like colloidal particles, ${ }^{5}$ its influence over the years has proven to be much more profound. The explanation of an ordered fluid formation, such as the nematic phase, as the result of the competition between orientational and translational entropy contributions, introduced the far reaching concept of "ordering entropy," while the idea of expressing the system's free energy as a functional of single particle density is a precursor of modern density functional theory (DFT) (e.g., Ref. 6).

The original Onsager theory accounted only for the second-virial coefficient contribution, thus (strictly) limiting its applicability to rod-like particle systems with large aspect ratios, but several improvements have been more recently proposed to overcome this drawback and include also

\footnotetext{
a) alberta.ferrarini@unipd.it

b) achille@unive.it

c) giorgio.cinacchi@uam.es
}

higher order contributions. This prompted a number of DFT approaches with different degrees of sophistication, as well as a series of computer simulations, ${ }^{7-9}$ that can be applied and extended to many systems, either mono- or poly-disperse, both homo- and hetero-geneous. All these studies have confirmed that entropy alone can lead to complex phase organisations, including smectic and columnar liquid crystals. Recent simulations have further unveiled examples of the zoo of morphologies that can be obtained from packing of particles of different shape. ${ }^{10,11}$

So far, most theoretical and computational studies have focussed on convex hard particles (e.g., Ref. 7), while concave particles have been given less attention. Besides simple dumbbells, these include bent-core, ${ }^{12,13}$ lens-like, ${ }^{14}$ and bowl-shaped ${ }^{15}$ particles. Somewhat surprisingly, hard helices are not part of the above list, in spite of the several examples of this shape that can be found in natural and synthetic polymers. Rigid and semiflexible helical polymers (polynucleotides, polypeptides, viruses) have a well known propensity to form liquid crystal phases at high concentration. ${ }^{16-19}$ When examining and interpreting the experimental phase behaviour, helicoidal particles were generally assimilated to rods, thus neglecting specific effects of the actual shape (e.g., Ref. 20).

In this work, we address this problem by undertaking a study of the phase behaviour of hard helices as a function of their structural features. The model helices, shown in Fig. 1, are obtained by considering a set of fused hard spheres all having diameter $D$, and arranged in a helical fashion along a string of contour length $L$. Helices of different shapes and Euclidean lengths $\Lambda$ are generated by tuning the radius $r$ and the pitch $p$ (the helix parameters are defined in Appendix A).

Considering systems of rigid homochiral helices, we have focussed on the effects of the particle shape on the IN 


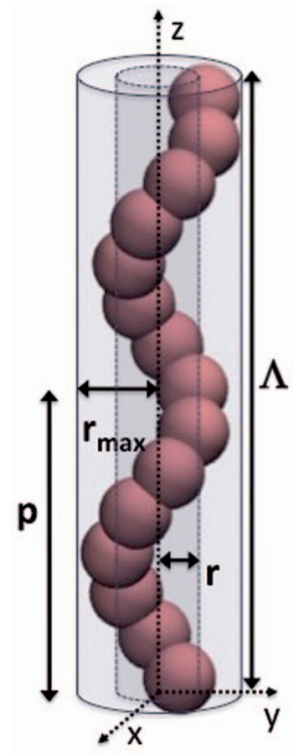

FIG. 1. Model helix and its characteristic parameters.

phase transition. Homochiral helices are expected to form a chiral nematic phase (cholesteric), in which the local preferred orientational axis (the director) rotates in space around a perpendicular axis. However, the cholesteric pitch is known to be orders of magnitude larger than length scale of interparticle interactions; thus the local phase properties are virtually indistinguishable from those of the corresponding untwisted nematic phase and the influence of chirality on the phase boundaries can be neglected at this stage. ${ }^{21}$ In addition, the presence of periodic boundary conditions in the Monte Carlo (MC) computations does not allow the emergence of an equilibrium cholesteric order, with a pitch much longer than the size of the simulation box. For these reasons, phase chirality will not be considered in the present study and will be the object of a future dedicated study.

Our approach hinges upon Onsager theory supported by MC simulations. We have used a simple extension of the Onsager theory suitable for particles with a finite aspect ratio, where the role of higher order virial terms is effectively taken into account. ${ }^{22,23}$ This theory was previously applied with success to the thermodynamics of the IN phase transition in hard, straight rods. ${ }^{23}$ Here its performance for the more subtle and challenging case of hard helices has been tested by comparison with MC simulations. As further elaborated below, $\mathrm{MC}$ simulations for hard helices are considerably more demanding with respect to the spherocylinder counterpart. Onsager theory, being computationally much less expensive, can then be useful for a preliminary exploration of the phase diagram.

Through a combined action of theory and simulations, we will provide evidence that the helical shape affects the location of the IN transition in comparison with hard rods of similar length and diameter, thus casting some doubts on the possibility of providing a straightforward link between the phase behaviour of hard helices and that of hard rods.

The layout of this work is as follows. In Sec. II our Onsager and MC approaches are illustrated, while theoretical and simulation results are presented and discussed in Sec. III.
Section IV concludes this work by summarising present findings and providing an outlook for future developments.

\section{THEORY AND SIMULATIONS}

\section{A. Theory}

\section{Free energy of the isotropic and nematic phases}

Let us consider a system of $N$ identical helices in a volume $V$ at temperature $T$. We denote by $v=V / N$ the volume per particle and $\eta=v_{0} / v$ the packing density, where $v_{0}$ is the volume of a particle.

The mutual interaction between a pair of hard helices (1 and 2) takes the form:

$$
U\left(\boldsymbol{R}_{12}, \boldsymbol{\Omega}_{12}\right)= \begin{cases}\infty & \text { if } 1,2 \text { overlap } \\ 0 & \text { if } 1,2 \text { do not overlap }\end{cases}
$$

where $\boldsymbol{R}_{1}$ and $\boldsymbol{R}_{2}$ are the positions of the center-of-mass for helices 1 and 2, respectively, $\boldsymbol{R}_{12}=\boldsymbol{R}_{2}-\boldsymbol{R}_{1}$ is a vector defining the relative position of helix 2 with respect to helix 1 and $\boldsymbol{\Omega}_{12}=\left(\alpha_{12}, \beta_{12}, \gamma_{12}\right)$ are the Euler angles that define the rotation from 1 to 2 .

In the Onsager approach the free energy of the system is expressed as a functional of the single particle density function $\rho(\boldsymbol{R}, \boldsymbol{\Omega})$, where $\boldsymbol{R}$ is the particle position and $\boldsymbol{\Omega}=(\alpha, \beta, \gamma)$ are the Euler angles specifying the particle orientation, with the normalization condition $\int d \boldsymbol{R} d \boldsymbol{\Omega} \rho(\boldsymbol{R}, \boldsymbol{\Omega})=N$. The nematic phase is uniform and the density function depends only on the particle orientation. Assuming helices as uniaxial particles, ${ }^{24}$ the single particle density in the uniaxial nematic phase (see below) reduces to $\rho=\rho(\beta)=f(\beta) /\left(4 \pi^{2} v\right)$, where $\beta$ is the angle between the helix axis and the nematic director and $f(\beta)$ is the orientational distribution function. The latter obeys the normalization condition $\int_{-1}^{1} d(\cos \beta) f(\beta)=1$ and is a constant equal to $1 / 2$ in the isotropic phase.

The free energy can then be expressed as

$$
\begin{aligned}
A[f(\beta)]= & N k_{B} T\left[\ln \frac{\Lambda_{\text {tr }}^{3}}{V} \frac{\Theta_{\text {or }}}{T}+\ln N-1\right] \\
& +A^{\text {or }}[f(\beta)]+A^{\mathrm{ex}}[f(\beta)] .
\end{aligned}
$$

The first term is the Helmholtz free energy of the ideal gas: $\Lambda_{\mathrm{tr}}=\left(h^{2} / 2 \pi k_{B} T m\right)^{1 / 2}$ is the de Broglie wavelength and $\Theta_{\text {or }}=h^{2} / 8 \pi^{2} k_{B} I$ is the rotational temperature, with $k_{B}$ and $h$ being the Boltzmann and the Planck constant, respectively, while $m$ is the mass and $I$ is the inertia moment of the particle. The second term in Eq. (2) accounts for the decrease of entropy due to orientational ordering:

$$
\frac{A^{\text {or }}}{N k_{B} T}=\int_{-1}^{1} d(\cos \beta) f(\beta) \ln [2 f(\beta)]
$$

and the last term, $A^{\text {ex }}$, represents the excess free energy. Within the Onsager formulation coupled with the Parsons-Lee (PL) correction, ${ }^{22,23}$ this is expressed in terms of the second virial contribution with a pre-factor, $G(\eta)$, that is meant to 
account for higher order virial contributions:

$$
\frac{A^{\mathrm{ex}}}{N k_{B} T}=\frac{G(\eta)}{2\left(4 \pi^{2}\right)^{2} v} \int d \boldsymbol{\Omega}_{1} f\left(\beta_{1}\right) \int d \boldsymbol{\Omega}_{2} f\left(\beta_{2}\right) v_{\mathrm{excl}}\left(\boldsymbol{\Omega}_{12}\right),
$$

where $v_{\text {excl }}\left(\mathbf{\Omega}_{12}\right)$ is the volume excluded to a helix (2) by another (1):

$$
v_{\text {excl }}\left(\boldsymbol{\Omega}_{12}\right)=-\int d \boldsymbol{R}_{12} e_{12}\left(\boldsymbol{R}_{12}, \boldsymbol{\Omega}_{12}\right),
$$

with the Mayer function: ${ }^{2}$

$$
e_{12}\left(\boldsymbol{R}_{12}, \boldsymbol{\Omega}_{12}\right)=\exp \left\{-U\left(\boldsymbol{R}_{12}, \boldsymbol{\Omega}_{12}\right) / k_{B} T\right\}-1 .
$$

Introducing the second virial coefficient:

$$
B_{2}=\frac{1}{2} \frac{1}{\left(4 \pi^{2}\right)^{2}} \int d \boldsymbol{\Omega}_{1} f\left(\beta_{1}\right) \int d \boldsymbol{\Omega}_{2} f\left(\beta_{2}\right) v_{\text {excl }}\left(\boldsymbol{\Omega}_{12}\right),
$$

the excess free energy Eq. (4) becomes

$$
A^{\mathrm{ex}}[f] / N k_{B} T=G(\eta) B_{2}[f] / v .
$$

\section{Parsons-Lee (PL) and modified Parsons-Lee (MPL) approximations}

The approximation proposed by Parsons ${ }^{22}$ and subsequently used by $\mathrm{Lee}^{23}$ and others ${ }^{25,26}$ for hard spherocylinders and ellipsoids relies on the assumption that the excess free energy is proportional to that of a system of hard spheres (HS) at the same packing fraction $(\eta)$ :

$$
\frac{A^{\mathrm{ex}}(\eta)}{N k_{B} T B_{2}(\eta)}=\frac{A_{H S}^{\mathrm{ex}}(\eta)}{N k_{B} T B_{2}^{H S}(\eta)} .
$$

Use of the Carnahan-Starling expression for the free energy of hard spheres, ${ }^{27}$ along with the relationships $B_{2}^{H S}=4 v_{H S}$ and $\eta=v_{H S} / v$, where $v_{H S}$ is the volume of a hard sphere, yields

$$
G(\eta)=\frac{A_{H S}^{\mathrm{ex}}(\eta)}{N k_{B} T B_{2}^{H S}(\eta)}=\frac{1}{4} \frac{4-3 \eta}{(1-\eta)^{2}} .
$$

In the original and subsequent works $s^{23,25,26}$ the volume of the reference hard spheres was taken equal to that of the spherocylinders or ellipsoids, $v_{H S}=v_{0}$. Good agreement between theory and simulations was obtained in that case, but significant discrepancies were found for linear particles made of tangentially bonded hard spheres. ${ }^{28}$ It has then been suggested that the assumption $v_{H S}=v_{0}$ may be inappropriate for hard non-convex bodies, since in this case the free volume available at a given number density is smaller than for convex particles having the same geometrical volume. ${ }^{29}$ It was proposed that in this case the volume of the reference hard spheres should be taken equal to an effective volume, $v_{\text {ef }}$, defined as the volume of the non-convex particle that is inaccessible to other particles. This effective volume is larger than the geometrical volume, and for linear chains of hard spheres it has been evaluated in Ref. 30 (see Appendix B). This variant of the PL theory has been given the name of modified ParsonsLee (MPL) theory. ${ }^{29}$

\section{Expansion in terms of orientational order parameters}

The orientational distribution function $f(\beta)$ is conveniently expanded over a basis of Legendre polynomials

$$
f(\beta)=\frac{1}{2} \sum_{j=0}^{\infty}(4 j+1)\left\langle P_{2 j}\right\rangle P_{2 j}(\cos \beta),
$$

where $\left\langle P_{2 j}\right\rangle$ are the nematic order parameters

$$
\left\langle P_{2 j}\right\rangle=\int_{-1}^{1} d(\cos \beta) f(\beta) P_{2 j}(\cos \beta) .
$$

The expansion is limited to polynomials of even rank in view of the nonpolar character of the nematic phase. The order parameters take values in the range $-1 / 2 \leq\left\langle P_{2 j}\right\rangle \leq 1$ and vanish in the isotropic phase.

Upon substituting Eq. (11) in Eqs. (3) and (4), and exploiting the properties of Wigner rotation matrices, ${ }^{31}$ we can express the orientational and excess contributions to the Helmholtz free energy as a function of the order parameters:

$$
\begin{aligned}
\frac{A^{\text {or }}}{N k_{B} T}= & \frac{1}{2} \sum_{j=0}^{\infty}(4 j+1)\left\langle P_{2 j}\right\rangle \int_{-1}^{1} d(\cos \beta) P_{2 j}(\cos \beta) \\
\times & \ln \left[\sum_{j^{\prime}=0}^{\infty}\left(4 j^{\prime}+1\right)\left\langle P_{2 j^{\prime}}\right\rangle P_{2 j^{\prime}}(\cos \beta)\right], \\
\frac{A^{\text {ex }}}{N k_{B} T}= & \frac{G(\eta)}{16 \pi^{2} v} \sum_{j=0}^{\infty}(4 j+1)\left\langle P_{2 j}\right\rangle^{2} \\
& \times \int d \boldsymbol{\Omega}_{12} P_{2 j}\left(\cos \beta_{12}\right) v_{\text {excl }}\left(\boldsymbol{\Omega}_{12}\right) .
\end{aligned}
$$

This leads to the following expression for the pressure

$$
\begin{aligned}
\frac{P}{k_{B} T}= & -\frac{1}{k_{B} T}\left(\frac{\partial A}{\partial V}\right)_{N T} \\
= & \frac{1}{v}+\frac{1}{16 \pi^{2} v^{2}}\left(G(\eta)+\frac{\eta(5-3 \eta)}{4(1-\eta)^{3}}\right) \\
& \times \sum_{j=0}^{\infty}(4 j+1)\left\langle P_{2 j}\right\rangle^{2} \int d \boldsymbol{\Omega}_{12} P_{2 j}\left(\cos \beta_{12}\right) v_{\mathrm{excl}}\left(\boldsymbol{\Omega}_{12}\right) .
\end{aligned}
$$

\section{B. Monte Carlo simulations}

In order to test the theoretical predictions, we implemented Isothermal-Isobaric (NPT) MC simulations ${ }^{32,33}$ on a system of $N$ hard helices, contained in cubic or orthorhombic computational boxes, with the usual periodic boundary conditions. Simulations were organised in cycles, each consisting of $2 N$ attempted particle moves (a random translation and rotation) and a volume move. Rotation trial moves were implemented either using the Barker-Watts ${ }^{34}$ or the quaternions methods, ${ }^{35,36}$ finding a good consistency between them. Volume moves were either performed scaling up or down the 


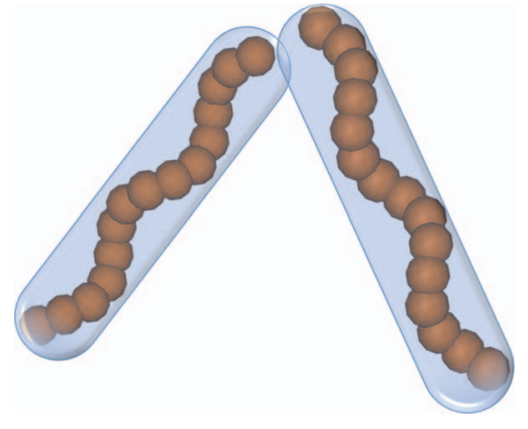

FIG. 2. Cartoon of the overlap between the spherocylinders containing a pair of helices.

box in those cases where cubic boxes were used or attempting to change a randomly selected edge of the box in the other cases. Being concerned with the IN phase transition only, we neglected from the outset all possible nuances necessary to properly account for other phases, such as twisted-nematic boundary conditions and variable-shape computational boxes.

The overlap condition was computed by first inserting each helix into the smallest spherocylinder containing it and testing for overlap between two such spherocylinders (see Fig. 2). This is a relatively fast test as it amounts to finding the minimal distance between two segments. To this purpose, we used the algorithm proposed by Vega and Lago. ${ }^{37}$ This method is approximatively four times faster than others previously used, essentially because it reduces to only four the number of regions to be checked for closest approach.

Only in the event of overlap between two spherocylinders, the spheres forming the embedded helices were tested for overlapping. This procedure significantly reduced the computational cost of the overlap test, that is one of the bottlenecks of this type of simulations, and considerably increased their efficiency.

The IN phase transition was monitored using the main orientational order parameter, $\left\langle P_{2}\right\rangle$, defined by Eq. (12) with $j=1$. To this aim, the following tensor ${ }^{38}$

$$
\mathbf{Q}_{\alpha \beta}=\frac{1}{N} \sum_{i=1}^{N} \frac{3}{2} \widehat{\mathbf{u}}_{i}^{\alpha} \widehat{\mathbf{u}}_{i}^{\beta}-\frac{1}{2} \delta_{\alpha \beta}
$$

was evaluated, and the corresponding eigenvalues and eigenvectors computed. Here $\alpha, \beta=x, y, z$, and $\widehat{\mathbf{u}}_{i}^{\alpha}$ is the $\alpha$ component of the unit vector $\widehat{\mathbf{u}}_{i}$ describing the orientation of the $i$ th helix axis. The orientational order parameter $\left\langle P_{2}\right\rangle$ was then identified with the largest eigenvalue of $\mathbf{Q}$. The difference between the other eigenvalues of $\mathbf{Q}$ was found to be smaller than $5 \%$, in agreement with our assumption of uniaxial nematic order.

\section{Computational details}

\section{Onsager theory calculations}

For each system, the Helmholtz free energy given in Eqs. (2) with Eqs. (13) and (14), was minimised at increasing values of the density $1 / v$, and the order parameters of the stable phase at each density value obtained are then used to calculate the pressure according to Eq. (15).

a. Evaluation of pair integrals. Integrals over all the relative positions and orientations of pairs of particles, appearing in Eqs. (14) and (15), were preliminarily evaluated and stored, to be used for the calculations at the various density values. These integrals have the general form:

$$
\begin{aligned}
& \int_{0}^{2 \pi} d \alpha_{12} \int_{-1}^{1} d\left(\cos \beta_{12}\right) P_{2 j}\left(\beta_{12}\right) \int_{0}^{2 \pi} d \gamma_{12} \int_{0}^{2 \pi} d \phi_{12} \\
& \quad \times \int_{-1}^{1} d\left(\cos \vartheta_{12}\right)\left(R_{12}^{0}\right)^{3}
\end{aligned}
$$

where $\mathbf{R}_{12}$, the vector position of helix 2 with respect to helix, is expressed in spherical coordinates, $\mathbf{R}_{12} \equiv\left\{R_{12}, \phi_{12}\right.$, $\left.\vartheta_{12}\right\}$ and $R_{12}^{0}$ is the closest approach distance, which is a function of the relative position and orientation of the two helices. The computational cost of this sixfold integral scales with $M^{2}$, where $M$ is the number of spheres in a helix. GaussLegendre and Gauss-Chebyshev quadrature algorithms were used to evaluate these integrals. ${ }^{39}$

b. Free energy minimization. It is expedient to choose as variational parameters the coefficients $u_{j}$ of the expansion

$$
-\ln f(\beta)=\sum_{j=1}^{\infty} u_{j} P_{2 j}(\cos \beta),
$$

rather than the order parameters. Equation (18) was used in Eq. (3) for the orientational contribution to the free energy, $A^{\text {or }}$, and was introduced into Eq. (14) for the excess contribution, $A^{\text {ex }}$, through the order parameters, Eq. (12). Thus, the Helmholtz free energy was expressed as a function of the $u_{j}$ coefficients. This has a twofold advantage: The expansion Eq. (18) converges faster than that of the density function (Eq. (11)) and the parameters $u_{j}$ are unconstrained, unlike order parameters.

\section{Monte Carlo simulations}

Our NPT MC simulations were carried out using $N$ $=675$ or 867 hard helices with periodic boundary conditions. As a general rule, we started a series of simulations from a diluted configuration and reached equilibrium upon compression. Typical equilibration runs consisted of $3 \times 10^{6} \mathrm{MC}$ cycles and were followed by a production run of additional $3 \times 10^{6} \mathrm{MC}$ cycles, during which averages of density and order parameter were calculated.

In most of the equilibration runs the maximum values for the displacement, rotation, and volume moves were varied in the course of the run to reach a $30 \%-40 \%$ of acceptance. This procedure is known to lead to a possible violation of the detailed balance condition, ${ }^{40}$ but we explicitly verified that this does not lead to any bias in the present case. During production runs, the overall acceptance ratio was adjusted to be $30 \%-40 \%$ by a suitable choice of the maximum displacement, rotation, and volume parameters, and these values were never altered during the run. 


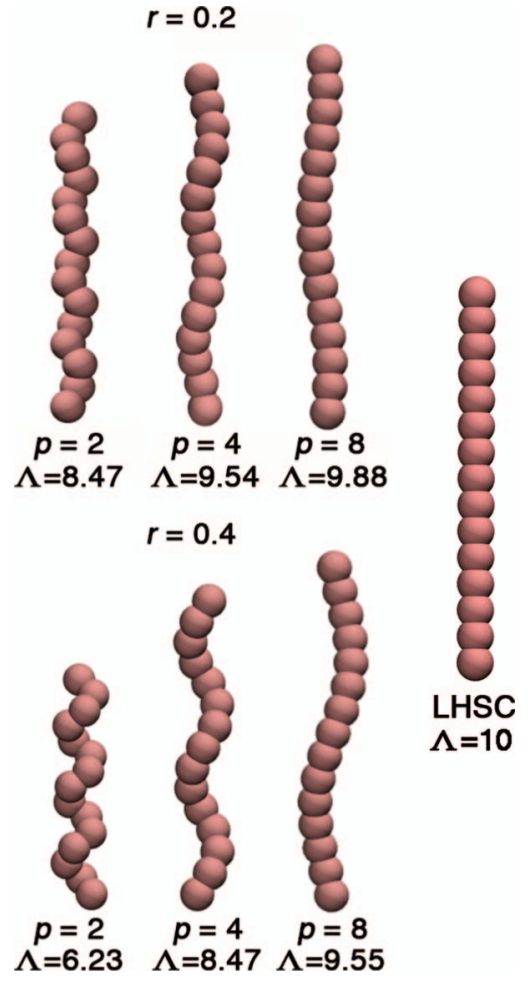

FIG. 3. Helices of radius $r$ and pitch $p$ investigated here. Helices have the same contour length $L$ but different Euclidean lengths $\Lambda$ (lengths are scaled with the sphere diameter $D$ ). For comparison also the fully extended linear hard sphere chain (LHSC) is shown.

It is worth emphasizing that simulations for hard helices are considerably more demanding from the computational point of view than simulations of hard spherocylinders. Depending on the state point considered and the values used for the radius and pitch, the computational cost might be as high as 8 times that of the corresponding spherocylinders.

\section{RESULTS AND DISCUSSION}

We have considered helical particles with different structural parameters (see Appendix A for the definition), as in Fig. 3. Helices are formed by 15 fused spheres of diameter D and have the same contour length $L=10 D$, but different pitches and radii.

In presenting and discussing our results we will use reduced units, with the diameter $D$ taken as the unit of length, and with reduced pressure $P^{*}=P D^{3} / k_{B} T$. For each system, the results from $\mathrm{MC}$ simulations will be compared with those from Onsager theory with the PL and the MPL approximation, which differ in the definition of the packing fraction entering the scaling factor $G$, Eq. (10): $\eta=v_{0} / v$, with $v_{0}$ being the geometric volume of the helix (PL), and $\eta=v_{e f} / v$, where $v_{e f}$ is the effective volume defined in Appendix B (MPL). Values of geometric and effective volume are reported in Tables I and II, respectively. MC data will be reported with error bars, evaluated according to the reblocking algorithm described in Ref. 41.

As a preliminary test, we have performed calculations for the LHSC, for which $\Lambda=L$. Figure 4 shows order parameter $\left\langle P_{2}\right\rangle$ and reduced pressure $P^{*}$ calculated for the LHSC as
TABLE I. Geometric volume $v_{0}$ of fused hard sphere helices of radius $r$ and pitch $p$, calculated using Eq. (B1). For comparison, also the value for LHSCs is reported.

\begin{tabular}{ccc}
\hline \hline Helix & $d_{c c}$ & $v_{0}$ \\
\hline$p=2, r=0.2$ & 0.687 & 6.89 \\
$p=2, r=0.4$ & 0.680 & 6.85 \\
$p=4, r=0.2$ & 0.711 & 7.02 \\
$p=4, r=0.4$ & 0.707 & 7.00 \\
$p=8, r=0.2$ & 0.714 & 7.04 \\
$p=8, r=0.4$ & 0.714 & 7.04 \\
LHSC & 0.714 & 7.04 \\
\hline \hline
\end{tabular}

a function of the packing fraction. At $\eta \sim 0.24$ an IN phase transition occurs, characterized by a jump in the order parameter. On moving deeper into the $\mathrm{N}$ phase $\left\langle P_{2}\right\rangle$ takes higher values, larger than 0.8 . The nonvanishing $\left\langle P_{2}\right\rangle$ obtained in the isotropic phase from simulations can be attributed to finitesize effects, and this feature is also present in the isotropic phase for helices. Figure 4 shows good agreement between theory and simulations for LHSCs. The results obtained using the PL and the MPL approximation are also very close one another, as expected in view of the high superimpositions of the spheres, so that the cavities between them have tiny volumes. This agrees with Ref. 29 where it was shown that for LHSCs the discrepancies between MC simulations and PL theory, and correspondingly also the improvements of the MPL scaling, decrease as the superimposition between adjacent spheres increases.

Figures 5-7 show order parameters and pressures calculated for the helices with $r=0.2$ and decreasing pitches $p=8,4$, and 2 . In all these cases an IN transition is clearly visible, with its location in density shifting from $\eta \sim 0.24$ to $\eta \sim 0.29$ with decreasing pitch from LHSC (infinite pitch) to the helix with shorter pitch $(p=2)$. This can be qualitatively understood in terms of the decrease of the Euclidean length (and hence the aspect ratio) with decreasing pitch. In all these cases, we find a good agreement between Onsager theory and numerical simulations in the location of the IN transition and in the density dependence of the $\left\langle P_{2}\right\rangle$ order parameter. However, pressure tends to be underestimated by theory, especially in the $\mathrm{N}$ phase, and these differences increase with increasing density and with decreasing pitch. The PL approximation does not appear to be adequate for these

TABLE II. Effective volume of fused hard sphere helices of radius $r$ and pitch $p$, calculated either by the program $\operatorname{MSMS}^{43}\left(v_{e f}\right)$ or using Eq. (B2) with the $d_{c c}$ distances reported in Table I $\left(v_{e f}^{L H S C}\right)$. For comparison, also the value for the LHSC is reported.

\begin{tabular}{ccc}
\hline \hline Helix & $v_{\text {ef }}$ & $v_{\text {ef }}^{\text {LHSC }}$ \\
\hline$p=2, r=0.2$ & 7.24 & 7.20 \\
$p=2, r=0.4$ & 7.78 & 7.15 \\
$p=4, r=0.2$ & 7.37 & 7.37 \\
$p=4, r=0.4$ & 7.34 & 7.34 \\
$p=8, r=0.2$ & 7.39 & 7.39 \\
$p=8, r=0.4$ & 7.39 & 7.39 \\
LHSC & 7.39 & 7.39 \\
\hline \hline
\end{tabular}



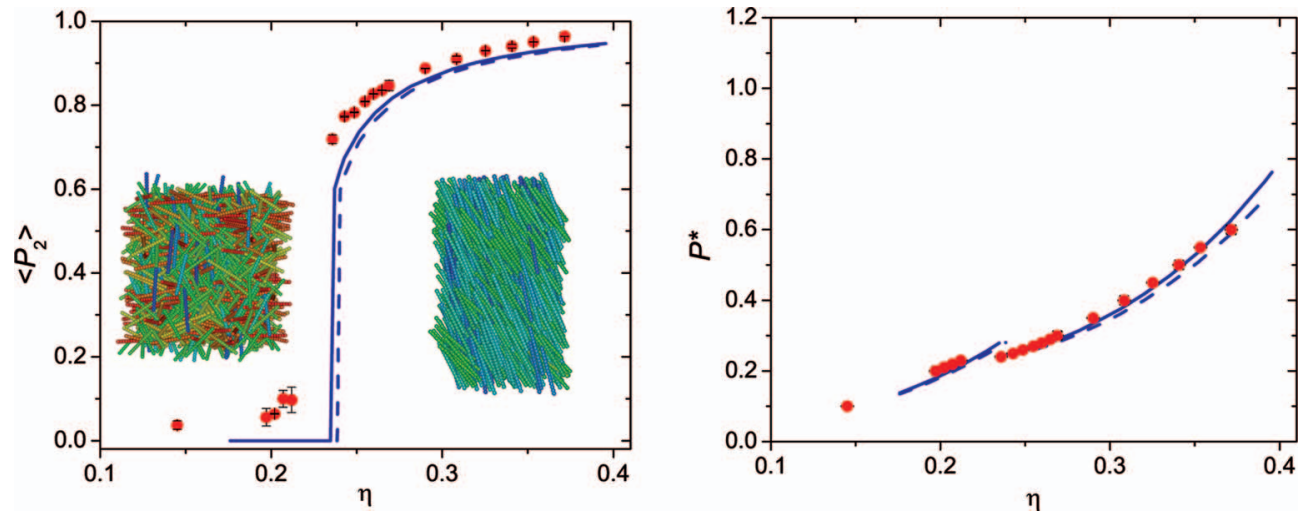

FIG. 4. $\left\langle P_{2}\right\rangle$ order parameter (Left) and reduced pressure $P^{*}$ (Right) as a function of the volume fraction $\eta=v_{0} / v$ for the LHSC, from MC simulations (closed circles) and from Onsager theory with PL (dashed line) or MPL (solid line) approximation. Insets on the left panel, here and in following figures, depict representative snapshots obtained using QMGA software. ${ }^{42}$
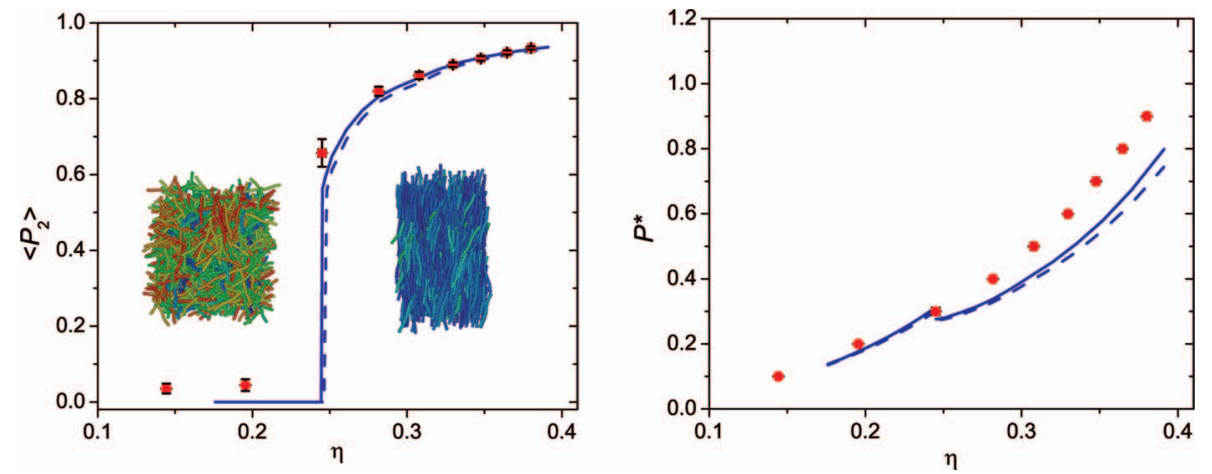

FIG. 5. $\left\langle P_{2}\right\rangle$ order parameter (Left) and reduced pressure $P^{*}$ (Right) as a function of the volume fraction $\eta=v_{0} / v$ for the helix with $p=8$ and $r=0.2$, from MC simulations (closed circles) and from Onsager theory with PL (dashed line) or MPL (solid line) approximation.
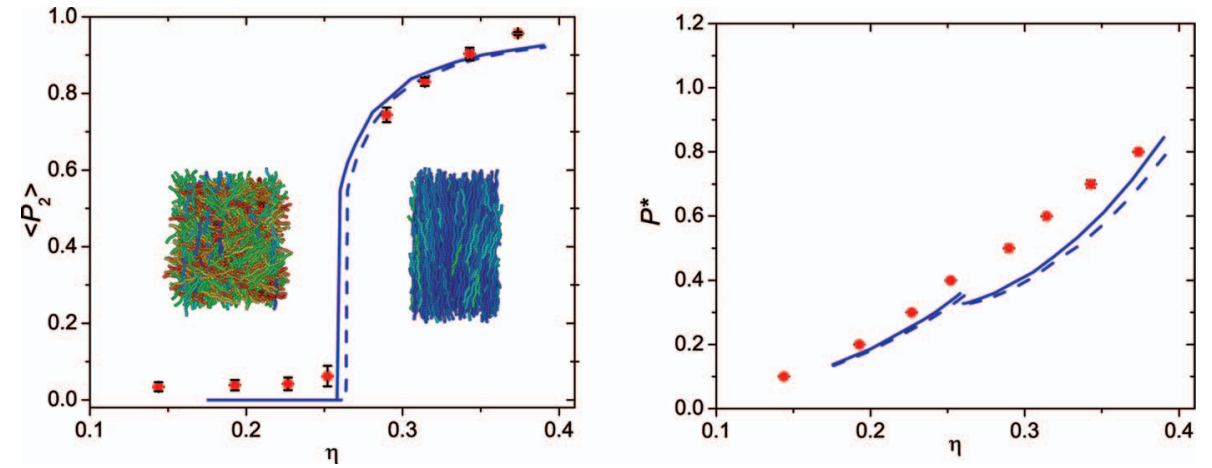

FIG. 6. $\left\langle P_{2}\right\rangle$ order parameter (Left) and reduced pressure $P^{*}$ (Right) as a function of the volume fraction $\eta=v_{0} / v$ for the helix with $p=4$ and $r=0.2$, from MC simulations (closed circles) and from Onsager theory with PL (dashed line) or MPL (solid line) approximation.
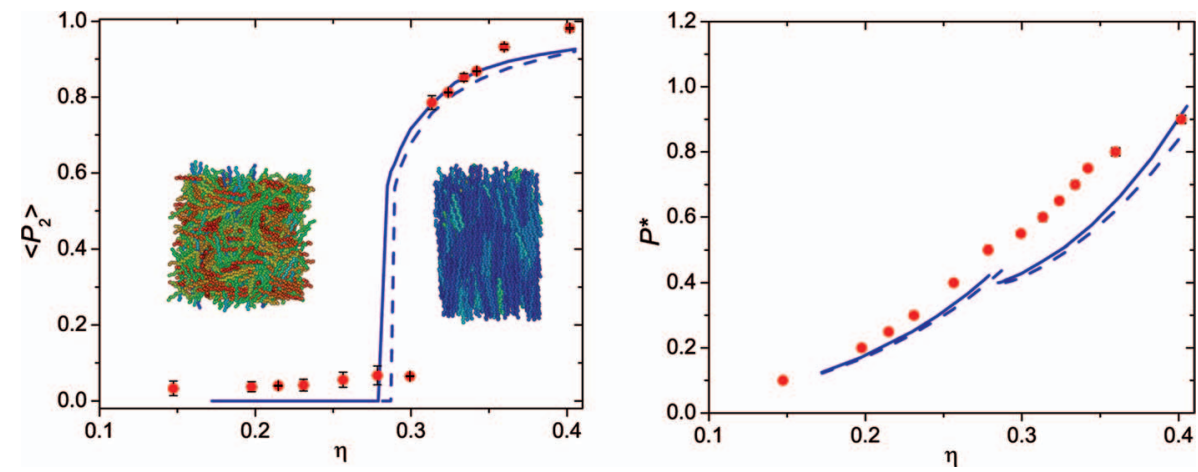

FIG. 7. $\left\langle P_{2}\right\rangle$ order parameter (Left) and reduced pressure $P^{*}$ (Right) as a function of the packing fraction $\eta=v_{0} / v$ for the helix with $p=2$ and $r=0.2$, from MC simulations (closed circles) and from Onsager theory with PL (dashed line) or MPL (solid line) approximation. 

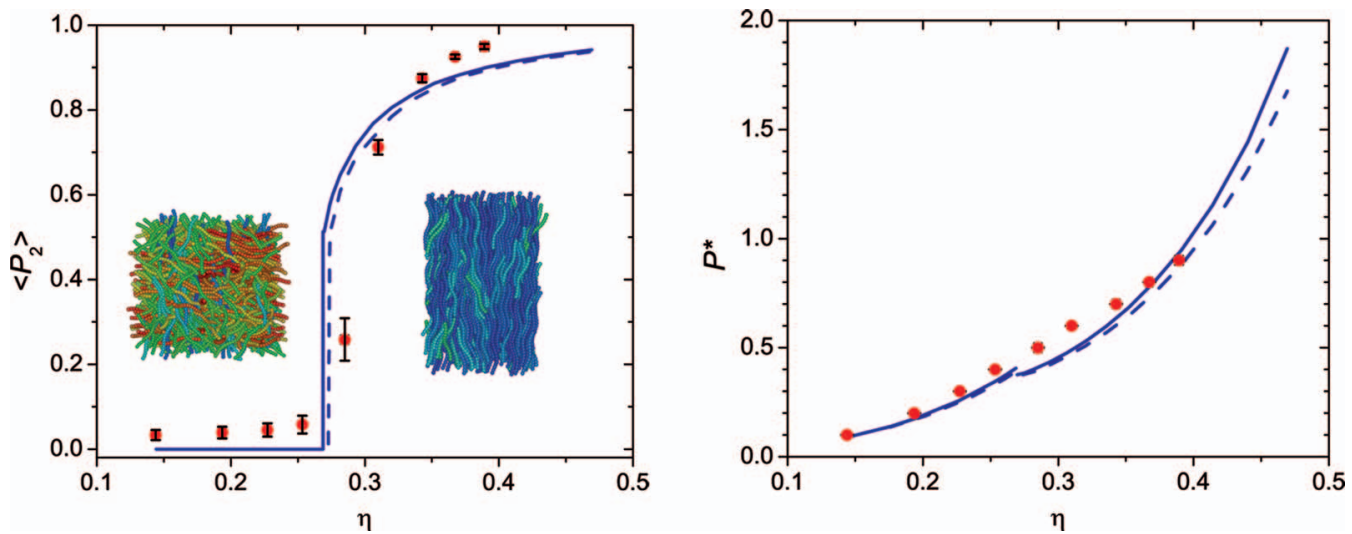

FIG. 8. $\left\langle P_{2}\right\rangle$ order parameter (Left) and reduced pressure $P^{*}$ (Right) as a function of the volume fraction $\eta=v_{0} / v$ for the helix with $p=8$ and $r=0.4$, from MC simulations (closed circles) and from Onsager theory with PL (dashed line) or MPL (solid line) approximation.

helical particles and use of the MPL variant leads only to a very slight improvement. The reason is that the non-convexity of the helices is not simply due to the voids between adjacent spheres (see Fig. 12), so removal of these voids is not sufficient to account for the real excluded volume.

The discrepancy with respect to LHSCs becomes even more pronounced for larger radii, as depicted in Figures 810 reporting the $\left\langle P_{2}\right\rangle$ order parameter and the reduced pressure calculated for helices with $r=0.4$ and $p=8,4$, and 2. These helices are curlier than those with smaller $r$ value (see Fig. 3), so it is not surprising that the differences from the behaviour of LHSCs are even more pronounced. No clear $\mathrm{N}$ phase is observed in simulations for the helices with $p=4$ and $p=2$, although at sufficiently high packing fraction $(\eta \approx 0.35)$ an anisotropic organization, with some signature of layered ordering, is visible. A complete characterization of these phases is delicate, mainly due to equilibration problems, and is presently under scrutiny. In the case of $p=8$ (see Fig. 8), a nematic phase was detected between $\eta \sim 0.27$ and $\eta \sim 0.38$; interestingly, the IN transition occurs at higher density than for the helices with smaller radius and similar Euclidean length $(r=0.2$ and $p=4)$. In all helices with $r=0.4$ we have also found a marked deviation between theoretical and $\mathrm{MC}$ results. In contrast to simulations, a nematic phase is predicted by Onsager theory for all pitch values, with the IN transition occurring at increasing density as the pitch decreases. Of course, being the theory implemented only for isotropic and uniaxial nematic phases, other possible phases could not be investigated. In short, only for the most elongated system ( $r=0.4$ and $p=8)$, we find a reasonable agreement between theory and simulations in this case. For shorter pitches, a jump in the $\left\langle P_{2}\right\rangle$ order parameter is obtained from simulations and theory at similar $\eta$ values, but the ordered phases appear to be different. As for pressure, differences between theory and simulations even appear in the isotropic phase for the helices with $p=4$ and $p=2$, with theoretical predictions lower than the MC results. For $p=2$ the improvement deriving from the MPL approximation is more significant than in the other cases, due to the larger value of the effective volume determined for this system using the rolling sphere criterion (see Table II and Appendix B).

An interesting last point, related to the above findings, is whether the IN phase transition for helices can be mapped on to that of rods in terms of simple parameters such as the aspect ratio, as generally done in experimental work on helical systems. ${ }^{20}$ Figure 11 collects the theoretical predic-
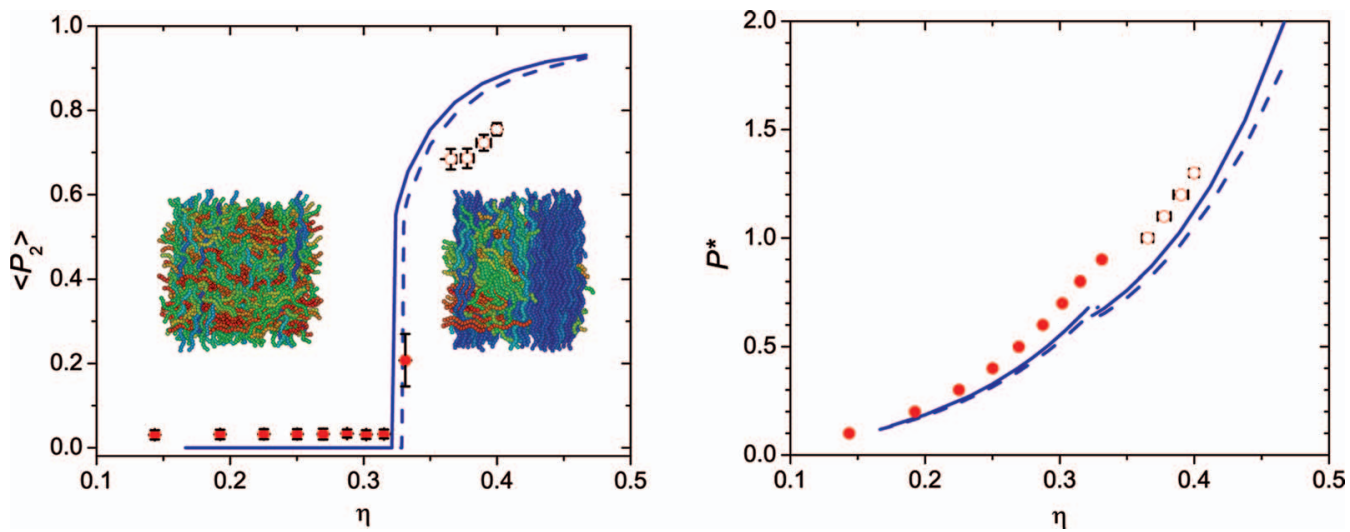

FIG. 9. $\left\langle P_{2}\right\rangle$ order parameter (Left) and reduced pressure $P^{*}$ (Right) as a function of the volume fraction $\eta=v_{0} / v$ for the helix with $p=4$ and $r=0.4$, from MC simulations (circles) and from Onsager theory with PL (dashed line) or MPL (solid line) approximation. Open circles are used for metastable states not yet fully characterized. 

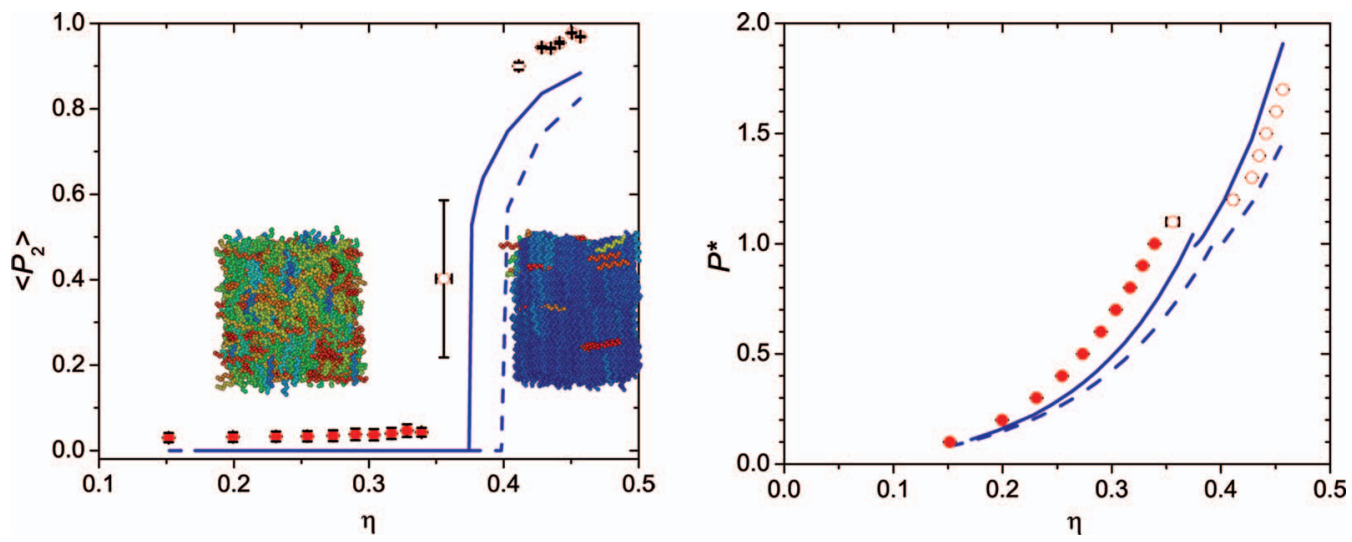

FIG. 10. $\left\langle P_{2}\right\rangle$ order parameter (Left) and reduced pressure $P^{*}$ (Right) as a function of the volume fraction $\eta=v_{0} / v$ for the helix with $p=2$ and $r=0.4$, from MC simulations (circles) and from Onsager theory with PL (dashed line) or MPL (solid line) approximation. Open circles are used for metastable states not yet fully characterized.

tions of the IN phase transition as a function the Euclidean length $\Lambda$. For comparison, the results obtained for LHSCs and those for spherocylinders are also reported. In the latter case the Onsager expression for the excluded volume was used. ${ }^{4}$ Of course the contour length $L$, which is identical for all the helices, is not a significant parameter in relation to the IN phase transition. On the other hand, Figure 11 also suggests that the Euclidean length, although more meaningful, is not fully satisfactory either, since for the same aspect ratio $\Lambda / D$, the density at which the IN transition occurs has a non-trivial dependence on the combination of the helical parameters $r$ and $p$. As a general rule, we find the transition to move towards higher volume fraction with increasing degree of nonconvexity. The fact that the location of the IN phase transition is not uniquely related to the aspect ratio may have implications for the analysis of experimental data for helical particles, as anticipated.

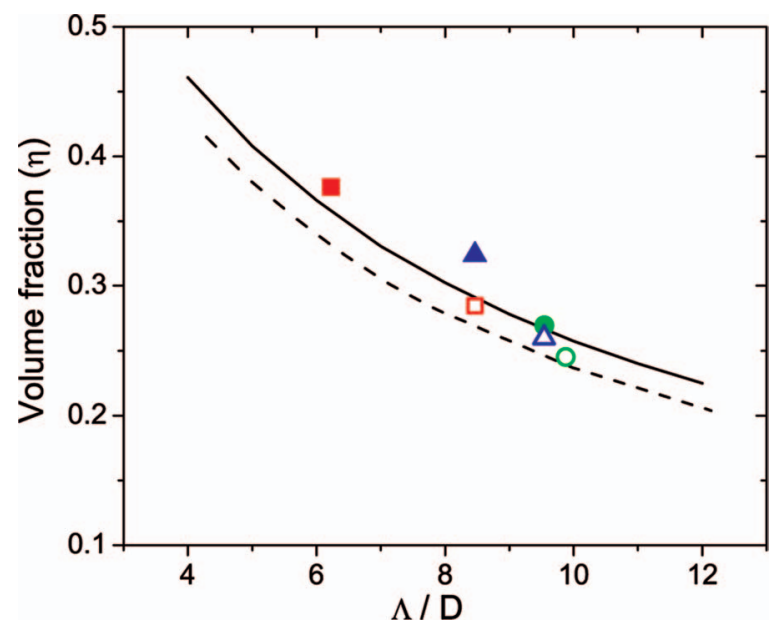

FIG. 11. Volume fraction $\eta=v_{0} / v$ at the isotropic-nematic transition as a function of the Euclidean length $(\Lambda)$, obtained from the Onsager theory with MPL approximation. Symbols refer to helices of different radius $r$ (open for $r=0.2$ and closed for $r=0.4$ ) and pitch $p$ (squares for $p=2$, triangles for $p=4$ and circles for $p=8$ ), lines are for LHSCs (dashed) and spherocylinders (solid).

\section{CONCLUSIONS}

In this work, systems of hard helical particles have been investigated using variations of Onsager theory and $\mathrm{MC}$ computer simulations. Our main goal was to rationalize the changes in the isotropic-to-nematic phase transition on going from straight rod-like to quite tortuous helical particles. We have found that helicity affects the location of the IN phase transition, with the latter in general being shifted to higher densities with increasing aspect ratios, as in spherocylinders. However, the same aspect ratio can be achieved with different structural parameters of hard helices, and this affects the IN phase transition. In other words, the aspect ratio alone cannot be considered as a good candidate for the interpretation of liquid-crystal phase diagrams of strongly curled helical particles. Our numerical results also unveiled the presence of additional ordered phases, especially in the case of highly distorted particles, that will require further analysis to assess their specific natures.

Another objective of our study was a test of Onsager theory for helical systems. We have examined whether the Onsager theory, which has proved to be successful in accounting for the thermodynamics of the IN phase transition in hard rods, can be extended to systems of helical non-convex particles. We have found that for high helicity Onsager theory significantly departs from numerical simulations, even when a modified form of the Parsons-Lee rescaling is included to account for the non-convexity of particles. When compared to the MC simulation data, Onsager theory generally underestimates pressure, with deviations that increase with increasing density and upon going from the isotropic to the nematic phase. This points to the need of a more effective theory for hard non-convex particles, a field that remains largely unexplored. Besides the Onsager theory employed here, various other theoretical approaches have been proposed, which include scaled-particle theory, ${ }^{44-46}$ the Vega and Lago theory that aims at incorporating a better description of the isotropic state, ${ }^{47}$ as well as Wertheim statistical mechanical treatment of associating fluids, ${ }^{48}$ which was successfully applied to bent-shaped particles in the isotropic phase. ${ }^{26}$ Another approach envisages the extension of the Onsager theory beyond 
the second virial contribution. We intend to undertake a thorough analysis and comparison of available theories in a future work.

This study is preliminary from several viewpoints and we plan to extend it in a number of ways, as alluded before. Though we dealt with chiral particles, phase chirality was not considered here, and the relationship between particle and phase chirality is one of the high priority points in our agenda. Other points that deserve close attention, and are currently under investigation, are a detailed numerical definition of the IN coexistence and the characterization of other phases occurring at higher densities. It will be of interest to compare these phases with those observed in experiments on helical flagella. ${ }^{49}$

\section{ACKNOWLEDGMENTS}

E.F. acknowledges the University of Padova for a scholarship (Ph.D. School in Materials Science and Engineering). H.B.K. acknowledges MIUR for a Ph.D. scholarship. A.F. and A.G acknowledge financial support from PRIN-COFIN20102011 (Contract No. 2010LKE4CC). G.C. is grateful to the Government of Spain for the award of a Ramón y Cajal research fellowship.

\section{APPENDIX A: DEFINITION OF FUSED HARD SPHERE HELICES}

A helix is made of $N$ spheres, whose centers are located at the points defined by the parametric equations:

$$
\left\{\begin{array}{l}
x_{i}=r \cos \left(2 \pi t_{i}\right) \\
y_{i}=r \sin \left(2 \pi t_{i}\right) \quad 1 \leq i \leq N, \\
z_{i}=p t_{i}
\end{array}\right.
$$

where $r$ is the radius and $p$ is the pitch of the helix (see Fig. 1). Given the values of $r, p$, and of the contour length $L$, the increment $\Delta t=t_{i+1}-t_{i}$ is determined by the equation:

$$
\frac{L}{N-1}=2 \pi \Delta t \sqrt{r^{2}+\left(\frac{p}{2 \pi}\right)^{2}} .
$$

The Euclidean length of the helix, defined as $\Lambda=z_{N}$ $-z_{1}$, depends on the pitch and radius, and coincides with the contour length $L$ only for $r=0$.

\section{APPENDIX B: MOLECULAR VOLUME AND EFFECTIVE VOLUME OF HELICES}

The volume $v_{0}$ of a linear chain formed by $m$ fused hard spheres (LHSC) of diameter $D$ and center-to-center distance $d_{c c}$ (see Fig. 12) is given by

$$
v_{0}=\frac{\pi}{6} D^{3}\left[1+\frac{m-1}{2}\left(3 \frac{d_{c c}}{D}-\left(\frac{d_{c c}}{D}\right)^{3}\right)\right]
$$

The same expression holds for a helix of fused hard spheres, provided that there are only two-sphere overlaps and the correct value of the distance $d_{c c}$ is used. ${ }^{50}$ For a given length of the curve connecting the centers of a pair of subsequent spheres, this distance depends on the helix radius and pitch.

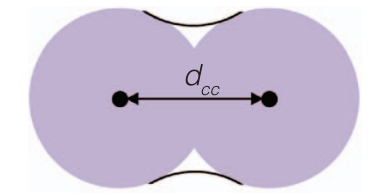

FIG. 12. Surface defining the effective volume of a pair of fused hard spheres.

Table I reports the (geometric) volume calculated for the all the helices shown in Fig. 3.

A definition of the effective volume has been proposed for LHSCs, as the volume enclosed by the surface drawn by a sphere identical to those of the chain, rolling over the particle. ${ }^{30}$ An example of this surface is shown in Fig. 12. The effective volume of the LHSC is then given by the expression:

$$
\begin{aligned}
v_{\mathrm{ef}}^{\mathrm{LHSC}}= & \frac{\pi}{6} D^{3}\left[1+(m-1)\left(3 \frac{d_{c c}}{D}-\frac{1}{2}\left(\frac{d_{c c}}{D}\right)^{3}\right.\right. \\
& \left.\left.-3 \sqrt{\left(1-\left(\frac{d_{c c}}{2 D}\right)^{2}\right)} \arcsin \left(\frac{d_{c c}}{2 D}\right)\right)\right] .
\end{aligned}
$$

We have adopted the same definition of the effective volume for fused hard sphere helices. However in this case, depending on the helix curvature, the effect of the rolling sphere can go beyond that of simply filling the voids between subsequent beads. We have calculated the effective volume $\left(v_{\text {ef }}\right)$ of helices using the program MSMS. ${ }^{43}$ The rolling sphere radius was taken equal to the radius of the fused hard spheres that form the helix. Table II reports the $v_{\text {ef }}$ values obtained for the helices shown in Fig. 3; for comparison we report in the table also the volume calculated according to Eq. (B2), using for each sphere the appropriate $d_{c c}$ value $\left(v_{\text {ef }}^{\text {LHSC }}\right.$ ). We can observe that $v_{\text {ef }}=v_{\text {ef }}^{\text {LHSC }}$ for all helices with longer pitch; only for $p=2$ there is some difference, more pronounced in the case with $r=0.4$. This discrepancy can be understood considering that these helices have grooves narrower than the sphere diameter $D$.

${ }^{1}$ J. A. Barker and D. Henderson, Rev. Mod. Phys. 48, 587 (1976).

${ }^{2}$ J. L. Barrat and J. P. Hansen, Basic Concepts for Simple and Complex Liquids (Cambridge University Press, Cambridge, 2003).

${ }^{3}$ M. N. Rosenbluth and A. W. Rosenbluth, J. Chem. Phys. 22, 881 (1954); B. J. Alder and T. E. Wainwright, ibid. 27, 1208 (1957).

${ }^{4}$ L. Onsager, Ann. N.Y. Acad. Sci. 51, 627 (1949).

${ }^{5}$ H. Zocher, Z. Anorg. Chem. 147, 91 (1925); F. C. Bawden et al., Nature (London) 138, 1051 (1936).

${ }^{6}$ D. Frenkel, Theor. Chem. Acc. 103, 212 (2000).

${ }^{7}$ M. P. Allen, G. T. Evans, D. Frenkel, and B. Mulder, Adv. Chem. Phys. 86, 1 (1993).

${ }^{8}$ G. J. Vroege and H. N. W. Lekkerkerker, Rep. Prog. Phys. 55, 1241 (1992).

${ }^{9}$ P. Tarazona, J. A. Cuesta, and Y. Martinez-Raton, Lect. Notes Phys. 753, 247 (2008)

${ }^{10}$ A. Haji-Akbari, M. Engel, A. S. Keys, X. Zheng, R. G. Petschek, P. Palffy-Muhoray, and S. C. Glotzer, Nature (London) 462, 773 (2009); P. Damasceno, M. Engel, and S. C. Glotzer, Science 337, 453 (2012).

${ }^{11}$ J. de Graaf, R. van Roij, and M. Dijkstra, Phys. Rev. Lett. 107, 155501 (2011).

${ }^{12}$ P. J. Camp, M. P. Allen, and A. J. Masters, J. Chem. Phys. 111, 9871 (1999).

${ }^{13}$ P. K. Maiti, Y. Lansac, M. A. Glaser, and N. A. Clark, Phys. Rev. Lett. 88, 065504 (2002); 92, 025501 (2004). 
${ }^{14}$ G. Cinacchi and J. S. van Duijneveldt, J. Phys. Chem. Lett. 1, 787 (2010).

${ }^{15}$ M. Marechal and M. Dijkstra, Phys. Rev. E 82, 031405 (2010).

${ }^{16}$ C. Robinson, Trans. Faraday Soc. 52, 571 (1956).

${ }^{17}$ R. Oldenbourg, X. Wen, R. B. Meyer, and D. L. D. Caspar, Phys. Rev. Lett. 61, 1851 (1988).

${ }^{18}$ F. Livolant and A. Leforestier, Prog. Polym. Sci. 21, 1115 (1996).

${ }^{19}$ I. W. Hamley, Soft Matter 6, 1863 (2010).

${ }^{20}$ M. Nakata, G. Zanchetta, B. D. Chapman, C. D. Jones, J. O. Cross, R. Pindak, T. Bellini, and N. A. Clark, Science 318, 1276 (2007).

${ }^{21} \mathrm{~S}$. Chandrasekhar, Liquid Crystals (Cambridge University Press, Cambridge, 1992).

${ }^{22}$ J. D. Parsons, Phys. Rev. A 19, 1225 (1979).

${ }^{23}$ S. D. Lee, J. Chem. Phys. 87, 4972 (1987); 89, 7036 (1988).

${ }^{24}$ Note that a finite helix has $C_{2}$ point symmetry, yet we have verified that the helices examined here have nearly uniaxial order (with the helix axis as the ordering axis) in the uniaxial nematic phase.

${ }^{25}$ S. C. McGrother, D. C. Williamson, and G. Jackson, J. Chem. Phys. 104, 6755 (1996).

${ }^{26}$ P. J. Camp, C. P. Mason, M. P. Allen, A. A. Khare, and D. A. Kofke, J. Chem. Phys. 105, 2837 (1996).

${ }^{27}$ N. F. Carnahan and K. E. Starling, J. Chem. Phys. 51, 635 (1969).

${ }^{28}$ D. C. Williamson and G. Jackson, J. Chem. Phys. 108, 10294 (1998).

${ }^{29}$ S. Varga and I. Szalai, Mol. Phys. 98, 693 (2000).

${ }^{30}$ J. L. F. Abascal and S. Lago, J. Mol. Liq. 30, 133 (1985).

${ }^{31}$ A. D. Varshalovich, N. A. Moskalev, and V. K. Kersonskii, Quantum Theory of Angular Momentum (World Scientific, New York, 1995).
${ }^{32}$ H. F. King, J. Chem. Phys. 57, 1837 (1972).

${ }^{33}$ W. W. Wood and J. D. Jacobson, J. Chem. Phys. 27, 1207 (1957).

${ }^{34}$ J. A. Barker and R. O. Watts, Chem. Phys. Lett. 3, 144 (1969)

${ }^{35}$ M. P. Allen and D. J. Tildesley, Computer Simulation of Liquids (Clarendon Press, Oxford, 1987)

${ }^{36}$ D. Frenkel and B. Smit, Understanding Molecular Simulation: From Algorithms to Applications (Academic, San Diego, 2002).

${ }^{37}$ C. Vega and S. Lago, Comput. Chem. 18, 55 (1994).

${ }^{38}$ J. Veillard-Baron, Mol. Phys. 28, 809 (1974).

${ }^{39}$ W. H. Press, B. P. Flannery, S. A. Teukolsky, and W. T. Vettering, Numerical Recipes (Cambridge University Press, Cambridge, 1986).

${ }^{40}$ M. A. Miller, L. M. Amon, and W. P. Reinhardt, Chem. Phys. Lett. 331, 278 (2000)

${ }^{41}$ H. Flyvbjerg and H. G. Petersen, J. Chem. Phys. 91, 461 (1989).

${ }^{42}$ A. T. Gabriel, T. Meyer, and G. Germano, J. Chem. Theory Comput. 4, 468 (2008), see http://qmga.sourceforge.net.

${ }^{43}$ M. F. Sanner, A. J. Olson, and J. C. Spehner, Biopolymers 38, 305 (1996).

${ }^{44}$ M. A. Cotter, J. Chem. Phys. 66, 1098 (1977).

${ }^{45}$ T. Boublik, C. Vega, and M. Diazpena, J. Chem. Phys. 93, 730 (1990).

${ }^{46}$ T. Jiang and J. Wu, J. Chem. Phys. 127, 034902 (2007).

${ }^{47}$ C. Vega and S. Lago, J. Chem. Phys. 100, 6727 (1994).

${ }^{48}$ M. S. Wertheim, J. Chem. Phys. 85, 2929 (1986).

${ }^{49}$ E. Barry, Z. Hensel, Z. Dogic, M. Shribak, and R. Oldenbourg, Phys. Rev. Lett. 96, 018305 (2006)

${ }^{50} d_{c c}=\sqrt{\left(x_{i+1}-x_{i}\right)^{2}+\left(y_{i+1}-y_{i}\right)^{2}+\left(z_{i+1}-z_{i}\right)^{2}}$ is the distance between centres of two subsequent spheres. 